\title{
Distribution of Recombination Currents in the Space Charge Region of Heterostructure Bipolar Devices
}

\author{
J. Pallarès, Student Member, IEEE, L. F. Marsal, Student Member, IEEE, \\ X. Correig, J. Calderer, and R. Alcubilla, Member, IEEE
}

\begin{abstract}
This paper addresses the problem of the space charge region Shockley-Read-Hall (SRH) recombination currents in heterojunctions with one noncrystalline side. A formulation which generalizes previous works is discussed. The approach is based on the drift-diffusion model with a thermionic-field emission boundary condition. The main physical parameters which determine the relative contribution of each zone of the space charge region (SCR) to the total recombination current are identified. The general analysis is applied for the first time to amorphous/crystalline heterojunctions and design criteria are established to minimize the total recombination current.
\end{abstract}

Index Terms - Amorphous semiconductor devices, semiconductor device modeling, semiconductor heterojunctions, space charge.

\section{INTRODUCTION}

A MORPHOUS/CRYSTALLINE and microcrystalline/ crystalline heterojunctions have been proposed as promising candidates for improving device performances. In particular, a-Si:H/c-Si [1], [2], a-SiC:H/c-Si [3], $\mu \mathrm{c}-\mathrm{Si} / \mathrm{c}-\mathrm{Si}$ [4], $\mu \mathrm{c}-\mathrm{Si} / \mathrm{a}-\mathrm{SiC}_{x} / \mathrm{c}-\mathrm{Si}[5]$, and $\mu \mathrm{c}-\mathrm{SiC} / \mathrm{c}-\mathrm{Si}$ [6] have been used as injector junctions in heterojunction bipolar transistors (HBT). However, experimentally measured base currents are usually higher than theoretically expected and most of the benefits of the heterojunction are lost.

Different recombination mechanisms may exist both in the space charge region (SCR) and in the neutral regions of a noncrystalline-crystalline heterojunction and contribute to the total forward current: radiative recombination, Auger recombination, Shockley-Read-Hall (SRH) recombination, etc. However an accurate analysis of the published $I-V$ characteristics suggests that the SRH recombination in the SCR is very often the dominant mechanism [7]. This recombination current mainly originates from the noncrystalline side of the junction where, in addition to interface states, there is a continuous trap distribution.

Consequently there is a need of an in-depth understanding of the SCR recombination currents in noncrystalline/crystalline heterojunctions in order to find the conditions under which such a current may be minimized. Two main questions should

Manuscript received May 14, 1997; revised September 3, 1997. This work was supported by the Comisión Interministerial de Ciencia y Tecnología (CICYT), Program TIC 96-1058.

J. Pallarès, L. F. Marsal, and X. Correig are with the Departament d'Enginyeria Electrònica, Universitat Rovira i Virgili, 43006 Tarragona, Spain.

J. Calderer and R. Alcubilla are with the Departament d'Enginyeria Electrònica, Universitat Politècnica de Catalunya, 08034 Barcelona, Spain.

Publisher Item Identifier S 0018-9383(98)00266-4. be addressed: first, how should the classical theory of the SRH recombination in the SCR be modified when there is a continuous trap distribution within the gap; second, how can the parameters of the heterojunction (bandgaps, doping concentrations) modify the relative contribution of each part of the SCR to the total recombination current in noncrystalline/crystalline heterojunctions. We have dealt with the first point in a recent work [8] and we now turn to the second one. The objective, from a design point of view, is to find criteria for minimizing the total SCR recombination current in a noncrystalline/crystalline heterojunction while at the same time discussing the restrictions introduced in the field of device applications.

Generation-recombination currents in the SCR of a P$\mathrm{N}$ junction were first analyzed by Sah et al. [9]. In this classical work, analytical expressions were derived by integrating the SRH recombination rate [10], [11] assuming one single level trap located at midgap. This leads to the well-known $\exp \left(V / 2 V_{T}\right)$ current dependence. The classical theory was extended by Choo to an asymmetrical P-N junction [12]. In this work, the effects on the recombination rate of the doping concentrations, capture cross section, asymmetry and energy level of the trap were analyzed but limited to a single level. However neither the ideality factor nor the activation energy of the saturation current were analyzed. We recently studied the SRH recombination currents of an asymmetrical P-N homojunction in the presence of discrete or continuous distributions of recombination centers [8]. For a single level trap analytical expressions were obtained showing that, depending on the trap energy level within the forbidden band, the ideality factor might be 2 or 1 and the energy activation of the saturation current moves from $E_{g} / 2$ to $E_{g}$.

On the other hand, the work on noncrystalline/crystalline heterojunctions has been mainly experimental. Up to now the most successful results in silicon wide gap emitter HBT's have been obtained using $\mu \mathrm{c}-\mathrm{Si} / \mathrm{a}-\mathrm{Si}_{1-x} \mathrm{C}_{x}$ as the emitter, with a current gain of 523 [4] and a cutoff frequency of $47 \mathrm{GHz}$ [5]. The analysis of the experimental Gummel plots from [5] reveals that the base current is dominated by the recombination current in the emitter-base SCR. However, as yet there is no detailed study of the SRH recombination currents in the SCR of silicon wide gap emitter HBT's.

This work helps to fill the gap between the theoretical analysis of wide bandgap emitter silicon HBT and the experimental results. First, Section II discusses a theoretical formulation for the SRH recombination current in the SCR of an abrupt PN heterojunction which includes a thermionic-field 
emission boundary condition at the heterojunction. For crystalline heterojunctions, analytical expressions are derived by generalizing Searles et al. work [13] by including the Grinberg et al. formulation [14], allowing a hole quasi-Fermi level discontinuity [15] and assuming arbitrary single level trap energy inside the bandgap and different minority carrier lifetimes. The study is restricted to the SRH recombination current and neglects both radiative and Auger recombinations. In Section III, the key parameters which determine the relative magnitude of the SRH recombination current in each zone of the SCR are obtained. The effects of the doping level and the material parameters are studied separately in two specific cases. Finally, in Section IV, we apply the theoretical framework developed to an amorphous/crystalline abrupt heterojunction, and establish design criteria for minimizing SCR recombination current. We then use these criteria to discuss certain conditions which are necessary for noncrystalline/crystalline heterojunction design.

\section{CAlCUlation OF THE SRH CURRENTS}

The recombination-generation currents on each side of the PN heterojunction follow from

$$
\begin{aligned}
J_{\mathrm{SRH}} & =q \int_{-x_{\mathrm{n}}}^{0} R(x) d x+q \int_{0}^{x_{\mathrm{p}}} R(x) d x \\
& =J_{\mathrm{SRH}, \mathrm{N}}+J_{\mathrm{SRH}, \mathrm{P}}
\end{aligned}
$$

where the total width of the SCR is given by $W=x_{\mathbf{n}}+x_{\mathbf{p}}$.

In order to evaluate (1), an accurate expression of the recombination rate $R(x)$ is needed. Two different cases have been analyzed: 1) a single level trap in the gap, as is quite usual when dealing with crystalline materials, and 2) a continuous trap distribution for noncrystalline materials (in this work the $\mathrm{N}$ side). On the other hand, the inclusion of a generic abrupt PN heterojunction leads to possible discontinuities in the electron and hole quasi-Fermi levels $\Delta E_{f \mathbf{n}}$ and $\Delta E_{f \mathrm{p}}$, respectively, as depicted in Fig. 1 [15], [16] that should be known before evaluating $R(x)$. The presence of discontinuities in the quasi-Fermi levels prevents the drift-diffusion formulation from being used in the whole SCR. As it is usually done [17], we use the drift diffusion formulation in $\left(-x_{\mathrm{n}}, 0^{-}\right)$ and $\left(0^{+}, x_{\mathrm{p}}\right)$ linking both regions together with the current continuity stated in the boundary condition.

\section{A. Crystalline/Crystalline Heterojunctions}

In crystalline materials, recombination is assumed to be due to a dominant single level trap located at $E_{t}$ inside the bandgap. So, the SRH recombination rate can be written as [9]-[11]

$$
R\left(x, E_{t}\right)=\frac{p(x) n(x)-\mathrm{n}_{i}^{2}(x)}{\xi\left(x, E_{t}\right)+\tau_{\mathbf{n}}(x) p(x)+\tau_{\mathbf{p}}(x) n(x)}
$$

where $\tau_{\mathbf{n}}, \tau_{\mathrm{p}}$ are the electron and hole minority carrier lifetimes within the $\mathrm{SCR}, \mathrm{n}_{i}$ the intrinsic carrier concentration and $\mathrm{p}, \mathrm{n}$ are the free carrier concentrations. The term $\xi$ is defined as

$$
\begin{aligned}
\xi\left(x, E_{t}\right)= & 2 \mathrm{n}_{i}(x) \sqrt{\tau_{\mathbf{n}}(x) \tau_{\mathbf{p}}(x)} \\
& \cdot \cosh \left\{\left[\frac{E_{t}-E_{i}(x)}{k T}\right]+\ln \sqrt{\frac{\tau_{\mathbf{p}}(x)}{\tau_{\mathbf{n}}(x)}}\right\}
\end{aligned}
$$

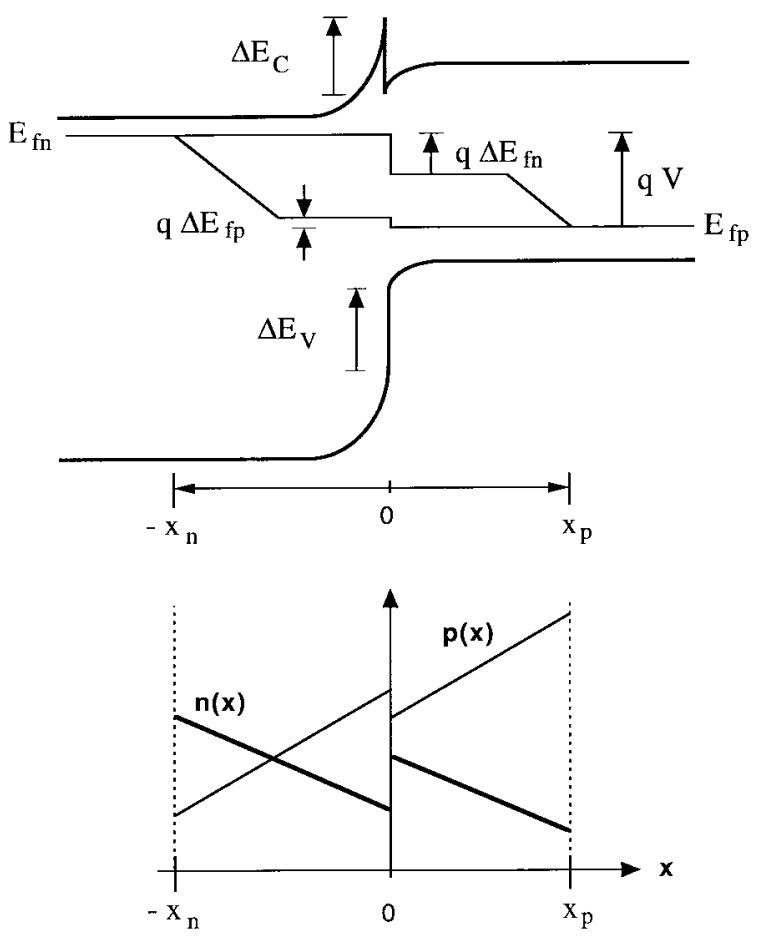

Fig. 1. Band diagram of an abrupt $\mathrm{N}$-large/P-small heterostructure under bias and a schematic free carrier concentration profile.

where $E_{i}$ is the intrinsic Fermi level, $k$ the Boltzmann constant, and $T$ the absolute temperature.

To obtain an analytical derivation, the electrostatic potential must be linearized [12] and the semiconductors assumed to be nondegenerate. The majority free carrier concentrations in each part of the SCR are expressed as

$$
\begin{aligned}
\mathrm{p}_{\mathrm{p}}(x)=\mathrm{N}_{A} \cdot \exp \left[\left(\frac{V-V_{\mathrm{bi}}}{V_{T}}\right)\left(\frac{x_{\mathrm{p}}-x}{W}\right)\right] \\
0<x<x_{\mathrm{p}} \\
\mathrm{n}_{\mathrm{n}}(x)=\mathrm{N}_{D} \cdot \exp \left[\left(\frac{V-V_{\mathrm{bi}}}{V_{T}}\right)\left(\frac{x_{\mathrm{n}}+x}{W}\right)\right] \\
-x_{\mathrm{n}}<x<0
\end{aligned}
$$

where $\mathrm{N}_{A}$ and $\mathrm{N}_{D}$ are the doping density concentration in the $\mathrm{P}$ and $\mathrm{N}$ region, respectively, $V_{T}=k T / q$ the thermal voltage, $V$ the applied voltage, and $V_{\mathrm{bi}}$ the built-in potential

$$
V_{\mathrm{bi}}=V_{T} \cdot \ln \left(\frac{\mathrm{N}_{A} \mathrm{~N}_{D}}{\mathrm{n}_{i, \mathrm{p}}^{2}} \cdot \frac{\mathrm{N}_{C}^{\mathrm{p}}}{\mathrm{N}_{C}^{\mathrm{n}}}\right)+\Delta E_{C}
$$

where $\mathrm{N}_{C}^{\mathrm{n}}$ and $\mathrm{N}_{C}^{\mathrm{p}}$ are the effective $\mathrm{N}$ - and P-side conduction band densities of states, respectively, and $\Delta E_{C}$ the conduction band discontinuity. Equation (6) is a generalization of the builtin potential expression proposed by Searles et al. (9) in [13]. Both equations are identical if $\mathrm{N}_{C}^{\mathrm{n}}=\mathrm{N}_{C}^{\mathrm{p}}$. In addition, (6) can be derived by integrating Grinberg's (9) [14] between the contacts under equilibrium conditions. Moreover, using the boundary condition at the heterointerface, which is given by (20) in [14], the minority free carrier concentration in the P-side of the SCR can be written as

$$
\mathrm{n}_{\mathrm{p}}(x)=\frac{\mathrm{n}_{i, \mathrm{p}}^{2}}{\mathrm{p}_{\mathrm{p}}(x)} \cdot \exp \left[\frac{V-\Delta E_{f \mathbf{n}}}{V_{T}}\right] \quad 0<x<x_{\mathrm{p}} .
$$


Parallel to the analysis done for electrons in [14], the following boundary condition can be derived for holes:

$$
\mathrm{p}_{(-)} \mathrm{n}_{(-)} \frac{\mathrm{N}_{V}^{\mathrm{n}}}{\mathrm{N}_{V}^{\mathrm{p}}} \frac{\mathrm{p}_{(+)}}{\mathrm{p}_{(-)}} \exp \left[-\frac{\Delta E_{V}}{V_{T}}\right]=\mathrm{n}_{i, \mathrm{n}}^{2} \exp \left[\frac{V}{V_{T}}\right]
$$

where $\Delta E_{V}$ is the valence band discontinuity, $\mathrm{n}_{(-)}$and $\mathrm{p}_{(-)}$ are, respectively, the electron and hole concentration in the $\mathrm{N}$-side near the $\mathrm{P}$-side junction, $\mathrm{p}_{(+)}$is the hole concentration on the P-side side of the abrupt boundary, and $\mathrm{N}_{V}^{\mathrm{n}}$ and $\mathrm{N}_{V}^{\mathrm{P}}$ are the $\mathrm{N}$-side and P-side effective valence band densities of states, respectively. From (8) we can obtain an expression for the built-in potential which is equivalent to (6). Also from (8) the minority free carrier concentration in the $\mathrm{N}$-side region of the SCR can be written as

$$
\mathrm{p}_{\mathbf{n}}(x)=\frac{\mathrm{n}_{i, \mathbf{n}}^{2}}{\mathrm{n}_{\mathbf{n}}(x)} \cdot \exp \left[\frac{V-\Delta E_{f \mathrm{p}}}{V_{T}}\right] \quad-x_{\mathbf{n}}<x<0 .
$$

Combining the set of equations (2)-(9), (1) can be analytically solved by using integral 2.451 \#4 in [18]. Then, we obtain (10a) and (10b) with (11a) and (11b) shown at the bottom of the page. The set of equations (10) and (11) give an analytical solution for $\mathrm{N}$-side and $\mathrm{P}$-side recombination currents in a generic PN crystalline/crystalline abrupt heterojunction.

Quasi-Fermi level discontinuities can be evaluated either through approximate analytical expressions [14], [15], [19] or exact numerical solutions using a current balancing at the heterointerface [13]. This method involves determining the tunneling factor $\gamma$, which can be calculated using the WKB approach which assumes the barrier to be triangular [17],
[20], [21]. This current balancing can be adopted as a general procedure for both quasi-Fermi level discontinuities.

Usually, for crystalline wide emitter HBT several additional assumptions are also made to simplify the final expressions: first, the recombination center is located exactly at midgap in the space-charge region, $E_{i}=E_{t}$; second, the electron and hole lifetimes within the SCR $\left(\tau_{y \mathrm{p}}=\tau_{y \mathrm{n}}\right)$ are constants; and third, the hole quasi-Fermi level discontinuity is neglected, $\Delta E_{f \mathrm{p}}=0$. In this particular case, the generic solution is reduced to Searles et al. model [13]

$$
\begin{aligned}
J_{\mathrm{SRH}, \mathrm{N}}(V)= & \frac{q \mathrm{n}_{i, \mathbf{n}}}{\tau_{\mathbf{n}}} \frac{W V_{T}}{V_{\mathrm{bi}}-V} \\
& \exp \left(\frac{V}{2 V_{T}}\right) \cdot \operatorname{atan}\left(\Psi_{\mathrm{N}}\right) \\
J_{\mathrm{SRH}, \mathrm{P}}(V)= & \frac{q \mathrm{n}_{i, \mathrm{p}}}{\tau_{\mathrm{p}}} \frac{W V_{T}}{V_{\mathrm{bi}}-V} \\
& \exp \left(\frac{V-\Delta E_{f \mathbf{n}}}{2 V_{T}}\right) \cdot \operatorname{atan}\left(\Psi_{\mathrm{P}}\right)
\end{aligned}
$$

with (13a) and (13b) shown at the bottom of the next page.

\section{B. Non-Crystalline/Crystalline Heterojunctions}

The effects of the heterojunction on the distribution of the recombination can be understood by analyzing crystalline heterojunctions. But, as we have pointed out in the introduction, knowing how the SCR recombination is distributed between the semizones is of practical interest mostly for devices where one side is noncrystalline.

$$
\begin{aligned}
& J_{\mathrm{SRH}, \mathrm{N}}(V)=\frac{q \mathrm{n}_{i, \mathbf{n}}}{\sqrt{\tau_{\mathrm{nn}} \tau_{\mathrm{pn}}}} \cdot \frac{W V_{T}}{V_{\mathrm{bi}}-V} \cdot \frac{\left[\exp \left(\frac{V-\Delta E_{f \mathrm{p}}}{V_{T}}\right)-1\right]}{\sqrt{\exp \left(\frac{V-\Delta E_{f \mathrm{p}}}{V_{T}}\right)-\cosh ^{2}\left[\left(\frac{E_{t}-E_{i, \mathbf{n}}}{k T}\right)+\ln \sqrt{\frac{\tau_{\mathrm{pm}}}{\tau_{\mathrm{nn}}}}\right]}} \cdot \operatorname{atan}\left(\Psi_{\mathrm{N}}\right) \\
& J_{\mathrm{SRH}, \mathrm{P}}(V)=\frac{q \mathrm{n}_{i, \mathbf{p}}}{\sqrt{\tau_{\mathrm{np}} \tau_{\mathrm{pp}}}} \cdot \frac{W V_{T}}{V_{\mathrm{bi}}-V} \cdot \frac{\left.\exp \left(\frac{V-\Delta E_{f \mathbf{n}}}{V_{T}}\right)-1\right]}{\sqrt{\exp \left(\frac{V-\Delta E_{f \mathbf{n}}}{V_{T}}\right)-\cosh ^{2}\left[\left(\frac{E_{t}-E_{i, \mathbf{p}}}{k T}\right)+\ln \sqrt{\frac{\tau_{\mathrm{pp}}}{\tau_{\mathrm{np}}}}\right.}} \cdot \operatorname{atan}\left(\Psi_{\mathrm{P}}\right)
\end{aligned}
$$

$$
\begin{aligned}
\Psi_{\mathrm{N}} & =\frac{\left\{\sqrt{\vartheta_{\mathrm{n}}^{2}(V)-\theta_{\mathrm{n}}^{2}\left(E_{t}\right)}\right\} \cdot \sinh \left[\varpi_{\mathbf{n}}(V)\right]}{\vartheta_{\mathbf{n}}(V) \cdot \cosh \left\{\varpi_{\mathbf{n}}(V)+\ln \left[\vartheta_{\mathbf{n}}(V) \cdot \frac{\mathrm{n}_{i, \mathbf{n}}}{\mathrm{N}_{D}} \cdot \sqrt{\frac{\tau_{\mathrm{pn}}}{\tau_{\mathrm{nn}}}}\right]\right\}+\theta_{\mathbf{n}}\left(E_{t}\right) \cdot \cosh \left[\varpi_{\mathbf{n}}(V)\right]} \\
\Psi_{\mathrm{P}} & =\frac{\left\{\sqrt{\vartheta_{\mathrm{p}}^{2}(V)-\theta_{\mathrm{p}}^{2}\left(E_{t}\right)}\right\} \cdot \sinh \left[\varpi_{\mathbf{p}}(V)\right]}{\vartheta_{\mathrm{p}}(V) \cdot \cosh \left\{\varpi_{\mathbf{p}}(V)+\ln \left[\vartheta_{\mathrm{p}}(V) \cdot \frac{\mathrm{n}_{i, \mathbf{p}}}{\mathrm{N}_{A}} \cdot \sqrt{\frac{\tau_{\mathrm{pp}}}{\tau_{\mathrm{np}}}}\right]\right\}+\theta_{\mathrm{p}}\left(E_{t}\right) \cdot \cosh \left[\varpi_{\mathbf{p}}(V)\right]} \\
\vartheta_{\mathbf{n}}(V) & =\exp \left(\frac{V-\Delta E_{f \mathbf{p}}}{2 V_{T}}\right) \vartheta_{\mathrm{p}}(V)=\exp \left(\frac{V-\Delta E_{f \mathbf{n}}}{2 V_{T}}\right) \tau_{y}=\sqrt{\tau_{\mathbf{n} y} \tau_{\mathbf{p} y}} \\
\varpi_{y}(V)= & \left(\frac{V_{\mathrm{bi}}-V}{2 V_{T}}\right) \frac{x_{y}}{W} \theta_{y}\left(E_{t}\right)=\cosh \left[\left(\frac{E_{t}-E_{i, y}}{k T}\right)+\ln \sqrt{\frac{\tau_{\mathbf{p} y}}{\tau_{\mathbf{n} y}}}\right] y=n, p
\end{aligned}
$$


In the noncrystalline region of the SCR, the recombination rate can be obtained by replacing (2) by [8]

$$
R(x)=\int_{0}^{E_{g}} \frac{g(E) \sigma_{\mathrm{n}}(x) \sigma_{\mathrm{p}}(x) v_{t h}\left[p(x) n(x)-\mathrm{n}_{i}^{2}(x)\right]}{\xi^{c}(x, E)+\sigma_{\mathrm{n}}(x) \cdot n(x)+\sigma_{\mathrm{p}}(x) \cdot p(x)} d E
$$

where $\sigma_{\mathbf{n}}$ and $\sigma_{\mathbf{p}}$ are the cross sections for electrons and holes, respectively, $v_{t h}$ is the thermal velocity, the term $\xi^{c}$ is defined as

$$
\begin{aligned}
\xi^{c}(x, E)= & 2 \mathrm{n}_{i}(x) \sqrt{\sigma_{\mathbf{n}}(x) \sigma_{\mathbf{p}}(x)} \\
& \cdot \cosh \left[\left(\frac{E-E_{i}}{k T}\right)+\ln \sqrt{\frac{\sigma_{\mathbf{n}}(x)}{\sigma_{\mathbf{p}}(x)}}\right]
\end{aligned}
$$

and $g(E)$ is the density of states inside the forbidden band. In the most generic form, $g(E)$ is written as a combination of uniform, exponential and Gaussian profiles [22], [23]

$$
\begin{aligned}
g(E)= & g_{u}+g_{a} \exp \left(\frac{E-E_{g}}{k T_{a}}\right)+g_{d} \exp \left(\frac{-E}{k T_{d}}\right) \\
& +\frac{\mathrm{N}_{d b}}{\sigma_{d b} \sqrt{2 \pi}} \exp \left[-\frac{\left(E-E_{d}\right)^{2}}{2 \sigma_{d b}^{2}}\right] .
\end{aligned}
$$

The free carrier distribution can be found in the same way as in the previous section. So, analytical closed forms are obtained only for recombination currents arising from the crystalline SCR side, while semi-analytical expressions are obtained for those arising from the noncrystalline SCR side.

\section{DISCUSSION}

In this section, the parameters which determine the distribution of the recombination current between both sides of the SCR of an abrupt heterojunction will be analyzed.

A simple but useful way of determining the relative magnitude of the SRH recombination currents arising from each SCR side is to define the quotient, because it indicates from which SCR side the total current arises. From (12) the ratio between the SRH recombination currents generated in the P-side and $\mathrm{N}$-side can be written as

$$
\frac{J_{\mathrm{SRH}, \mathrm{P}}}{J_{\mathrm{SRH}, \mathrm{N}}}=\frac{\mathrm{n}_{i, \mathrm{p}}}{\mathrm{n}_{i, \mathrm{n}}} \cdot \exp \left(-\frac{\Delta E_{f \mathrm{n}}}{2 V_{T}}\right) \cdot\left[\frac{\operatorname{atan}\left(\Psi_{\mathrm{P}}\right)}{\operatorname{atan}\left(\Psi_{\mathrm{N}}\right)}\right] .
$$

This ratio may be modified in two ways. First, by choosing appropriate materials for the heterojunction (e.g., in a Al$\mathrm{GaAs} / \mathrm{GaAs}$ heterojunction choose the Al fraction). Secondly,

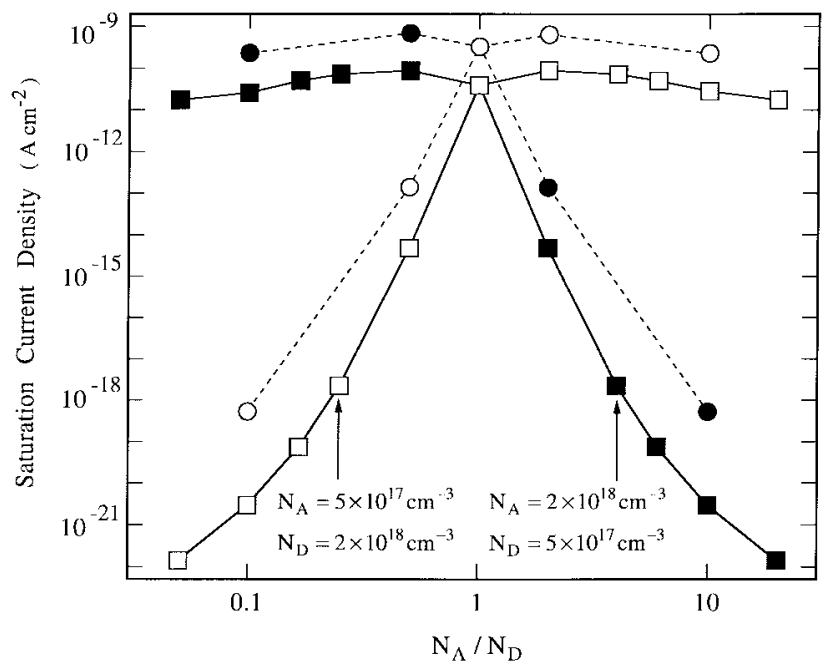

Fig. 2. Saturation current density versus $\mathrm{P}-\mathrm{N}$ doping ratio for the N-side (full symbol) and P-side (empty symbol) of a PN GaAs homojunction, for two doping references $5 \times 10^{17} \mathrm{~cm}^{-3}(\square)$ and $1 \times 10^{16} \mathrm{~cm}^{-3}(\bigcirc)$.

the quotient can in principle be tailored by modifying the doping concentrations which determine the SCR width, the built-in potential value and its distribution between both zones and the existence or not of discontinuities in the quasi-Fermi levels and their value.

Below both options will be analyzed separately. First we are going to qualify the effect of the doping concentration on the relative magnitude of the SRH recombination current arising from each side of the SCR. To isolate this variable we studied a GaAs homojunction. Subsequently, we will study the effect of the heterojunction together with the doping concentration on the distribution of the SCR SRH recombination current. To this end we will study the well-known AlGaAs/GaAs heterojunction.

\section{A. GaAs P-N Homojunction}

In order to isolate the effects of the doping level in (17), we first considered a PN GaAs abrupt homojunction diode. In this case, all the material parameters $\left(\tau, \mathrm{n}_{i}, \mathrm{~N}_{C}\right.$, and $\left.\mathrm{N}_{V}\right)$ are assumed to be constant and uniform throughout the SCR. Moreover, $\Delta E_{C}=\Delta E_{V}=\Delta E_{f \mathrm{n}}=0$. So, the current ratio is obtained directly from the term in brackets in (17).

Fig. 2 shows the saturation current densities obtained from each semizone separately as a function of the doping concentration ratio $\left(\mathrm{N}_{A} / \mathrm{N}_{D}\right)$ for two reference values, $\mathrm{N}_{r e f}$,

$$
\begin{aligned}
& \Psi_{\mathrm{N}}= \frac{\sinh \left[\left(\frac{V_{\mathrm{bi}}-V}{2 V_{T}}\right) \cdot \frac{x_{\mathrm{n}}}{W}\right]}{\cosh \left[\frac{V}{2 V_{T}}+\left(\frac{V_{\mathrm{bi}}-V}{2 V_{T}}\right) \cdot \frac{x_{\mathrm{n}}}{W}+\ln \left(\frac{\mathrm{n}_{i, \mathrm{n}}}{\mathrm{N}_{D}} \cdot \sqrt{\left.\left.\frac{\tau_{\mathrm{pn}}}{\tau_{\mathrm{nn}}}\right)\right]}\right.\right.} \\
& \Psi_{\mathrm{P}}=\frac{\sinh \left[\left(\frac{V_{\mathrm{bi}}-V}{2 V_{T}}\right) \cdot \frac{x_{\mathrm{p}}}{W}\right]}{\cosh \left[\left(\frac{V-\Delta E_{f \mathrm{n}}}{2 V_{T}}\right)+\left(\frac{V_{\mathrm{bi}}-V}{2 V_{T}}\right) \cdot \frac{x_{\mathrm{p}}}{W}+\ln \left(\frac{\mathrm{n}_{i, \mathrm{p}}}{\mathrm{N}_{A}} \cdot \sqrt{\frac{\tau_{\mathrm{pp}}}{\tau_{\mathrm{np}}}}\right)\right]}
\end{aligned}
$$


TABLE I

$\mathrm{Al}_{x} \mathrm{Ga}_{1-x}$ As/GaAs Parameters as a Function of the Aluminum Mole Fraction $x$

\begin{tabular}{lcc}
\hline \multicolumn{1}{c}{ Parameter } & Formula $(0 \leq \mathrm{x}<0.45)$ & Formula $(0.45 \leq \mathrm{x} \leq 1)$ \\
\hline Band gap (eV) & $1.424+1.247 \mathrm{x}$ & $1.9+0.125 \mathrm{x}+0.143 \mathrm{x}^{2}$ \\
Electron affinity (eV) & $4.07-1.1 \mathrm{x}$ & $3.46-0.14 \mathrm{x}$ \\
Electron effective mass & $(0.067+0.083 \mathrm{x}) \mathrm{m}_{\mathrm{o}}$ \\
Dielectric constant & $(13.18-3.12 \mathrm{x}) \varepsilon_{\mathrm{o}}$ \\
Electron and hole lifetime (s) & $10^{-9}$ \\
\hline
\end{tabular}

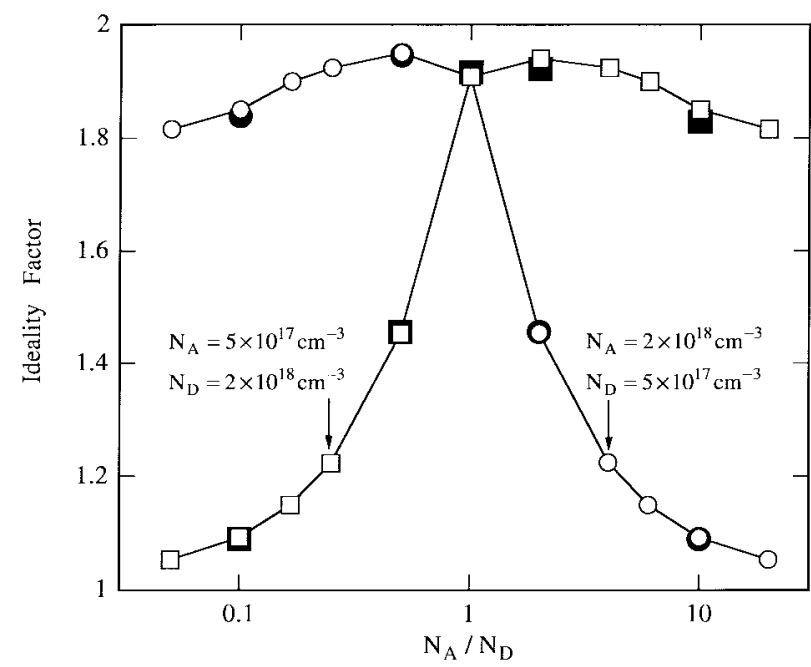

Fig. 3. Ideality factor versus P-N doping ratio for the $\mathrm{N}$-side ( $\square$ ) and P-side (O) of a PN GaAs homojunction, for two doping references $5 \times 10^{17} \mathrm{~cm}^{-3}$ (empty symbol) and $1 \times 10^{16} \mathrm{~cm}^{-3}$ (full symbol).

$1 \times 10^{16} \mathrm{~cm}^{-3}$ and $5 \times 10^{17} \mathrm{~cm}^{-3}\left(\mathrm{~N}_{\text {ref }}\right.$ is the value for which $\mathrm{N}_{A} / \mathrm{N}_{D}=1$ ). The total recombination current basically arises from the less doped side, whether it is the $\mathrm{N}$-side or the P-side. Moreover, the value obtained decreases with the doping reference value considered. The current arising from the highest doped side also decreases drastically with doping. It should be pointed out that this dependence is orders of magnitude stronger than is to be expected from the variation of the SCR width with the doping concentration.

Fig. 3 shows that the recombination current arising from the lowest doped side of the SCR has an ideality factor, $\eta$, close to 2 while the ideality factor of the current from the highest doped region tends to 1 as the doping ratio increases. Moreover, these values do not depend on the reference value chosen.

\section{B. AlGaAs/GaAs Heterojunction}

In this section a $(\mathrm{N}) \mathrm{Al}_{x} \mathrm{Ga}_{1-x} \mathrm{As} /(\mathrm{P}) \mathrm{GaAs}$ abrupt heterojunction is studied, but the conclusions obtained can be applied to other heterojunctions. In the design, we are free to change not only the doping ratio but also the material parameters, by modifying the aluminum mole fraction $x$. Table I shows the dependence on $x$ of the different parameters assumed in the calculations.

Two cases are discussed: $\mathrm{N}_{D} \gg \mathrm{N}_{A}$ and $\mathrm{N}_{A} \gg \mathrm{N}_{D}$.

1) $N_{D} \gg N_{A}$ : When the $\mathrm{N}$-side is assumed to be highly doped, $\Delta E_{f \mathrm{n}}$ is found to be negligible. Following Perlman

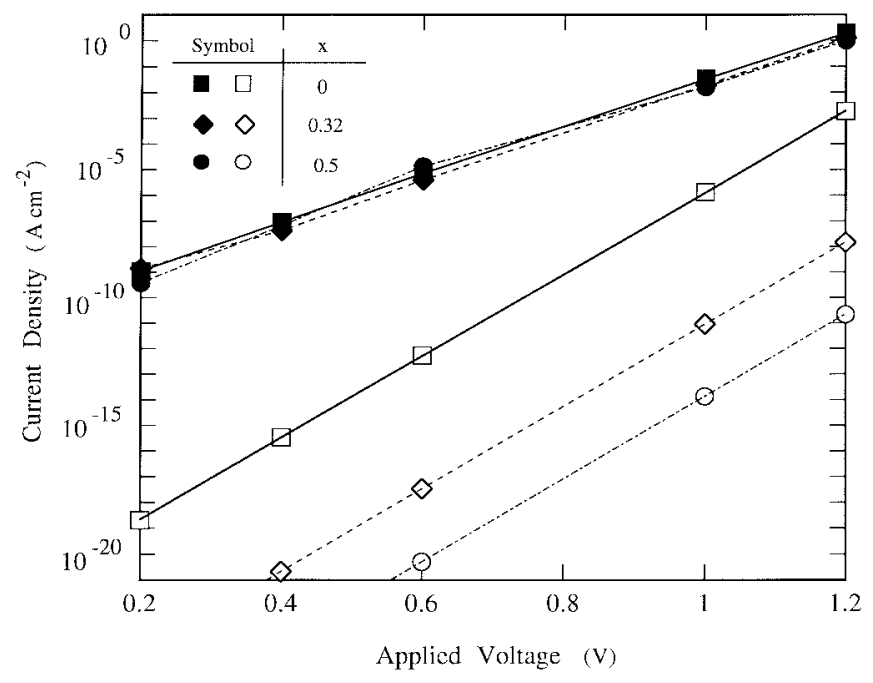

Fig. 4. N-side (empty symbol) and P-side (full symbol) $J-V$ characteristics of an $\mathrm{Al}_{x} \mathrm{Ga}_{1-x} \mathrm{As}(\mathrm{N}) / \mathrm{GaAs}(\mathrm{P})$ abrupt heterojunction, using $x$ as a parameter. The doping values are $\mathrm{N}_{A}=5 \times 10^{17} \mathrm{~cm}^{-3}$ and $\mathrm{N}_{D}=1 \times 10^{19} \mathrm{~cm}^{-3}$.

et al. [16], the band diagram has a negative reverse barrier and the heterojunction operates basically as a homojunction. Consequently, the recombination current is expected to arise from the P-side (less doped). As an example, Fig. 4 shows both components of the SRH current for $\mathrm{N}_{A}=5 \times 10^{17} \mathrm{~cm}^{-3}$ and $\mathrm{N}_{D}=1 \times 10^{19} \mathrm{~cm}^{-3}$, using the aluminum mole fraction as a parameter. The P-side current is constant with $x$, which means that the quasi-Fermi level discontinuity is negligible. On the other hand, the current from the $\mathrm{N}$-side decreases with $x$ because of the increase in the $\mathrm{N}$-side bandgap and the conduction band discontinuity. It can also be seen that, as in the homojunction, the largest component which originates from the lowest doped side has $\eta=2$ while the current from the highly doped $\mathrm{N}$ side has $\eta=1$.

2) $N_{A} \gg N_{D}$ : When the P-side doping is higher than the $\mathrm{N}$-side doping, as is typical in wide emitter GaAs-based HBT's, $\Delta E_{f \mathbf{n}}$ must be taken into account. In this case, the band diagram has a positive reverse barrier and the heterojunction operates as a metal-semiconductor junction [16]. Consequently, the recombination current is expected to arise from the $\mathrm{N}$-side (less doped) and thermionic emission and tunneling across the spike limit the total current. Fig. 5 shows the $I-V$ relation for the values $\mathrm{N}_{A}=1 \times 10^{19} \mathrm{~cm}^{-3}$ and $\mathrm{N}_{D}=5 \times 10^{17} \mathrm{~cm}^{-3}$. In this case, the current coming from the P-side (cathode) decreases with $x$ because $\Delta E_{f \mathbf{n}}$ increases. The current from the N-side of the SCR is the dominant term, and it also decreases with $x$ due to $E_{g}$ and $\Delta E_{C}$ effects. 


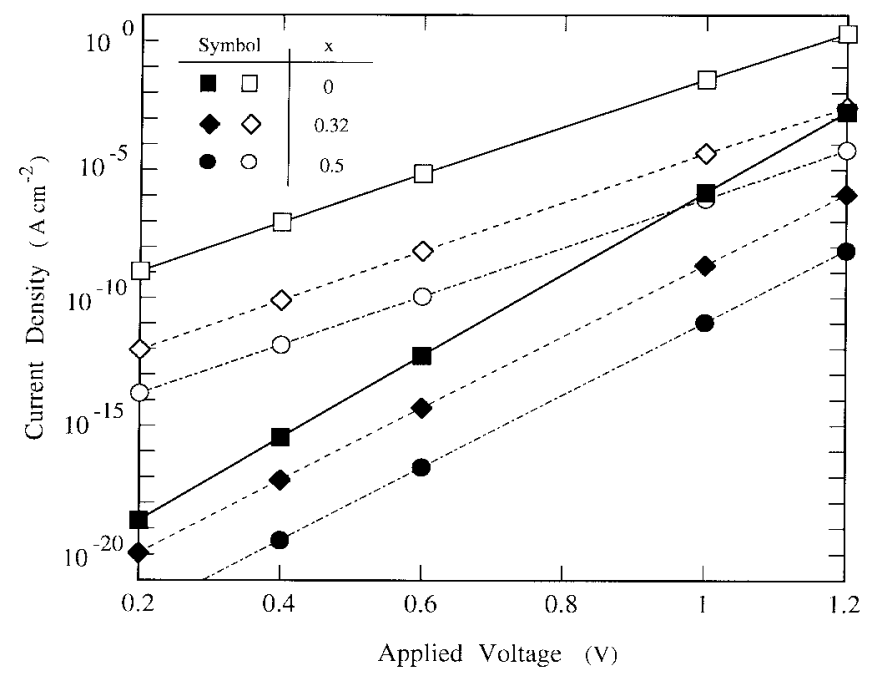

Fig. 5. N-side (empty symbol) and P-side (full symbol) $J-V$ characteristics of an $\mathrm{Al}_{x} \mathrm{Ga}_{1-x} \mathrm{As}(\mathrm{N}) / \mathrm{GaAs}(\mathrm{P})$ abrupt heterojunction, using $x$ as a parameter. The doping values are $\mathrm{N}_{A}=1 \times 10^{19} \mathrm{~cm}^{-3}$ and $\mathrm{N}_{D}=5 \times 10^{17} \mathrm{~cm}^{-3}$.

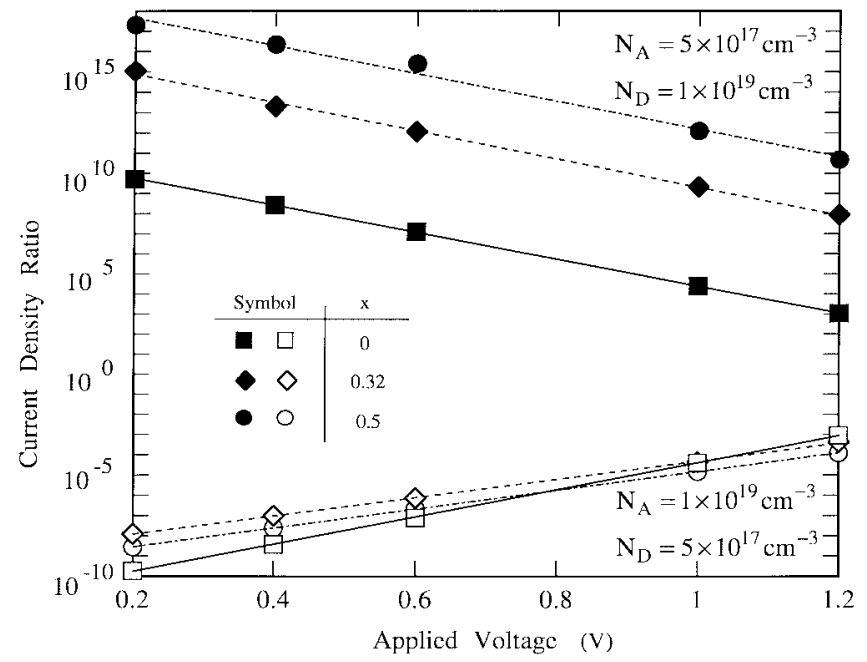

Fig. 6. The ratio of the $\mathrm{P}$-side/N-side recombination currents versus applied voltage, using the aluminum mole fraction $x$ of an $\mathrm{Al}_{x} \mathrm{Ga}_{1-x} \mathrm{As}(\mathrm{N}) / \mathrm{GaAs}(\mathrm{P})$ abrupt heterojunction as a parameter.

Finally, the ratio of the recombination currents arising from the $\mathrm{P}$ and $\mathrm{N}$ side is shown in Fig. 6, for two symmetrical doping concentrations. From this example it follows that by modifying the doping concentrations and the aluminum contents of the anode we determine in a large extent the SCR side where the recombination current will arise.

\section{APPLICATION TO \\ NONCRYSTALlinE/CRYSTALLINE HETEROJUNCTIONS}

The main interest in analyzing the relative contribution of each SCR side to the total recombination current in bipolar heterostructures is related to heterojunctions with noncrystalline material (amorphous or microcrystalline) on crystalline material. In these devices, the recombination current at the noncrystalline side is intrinsically large due to the high density of states in the bulk and the interface.

As an example, Fig. 7 shows, that for an (N)a-Si:H/(P)c-Si heterojunction diode amorphous material increases the $\mathrm{N}$-side recombination current and decreases the $\mathrm{P}$-side recombination

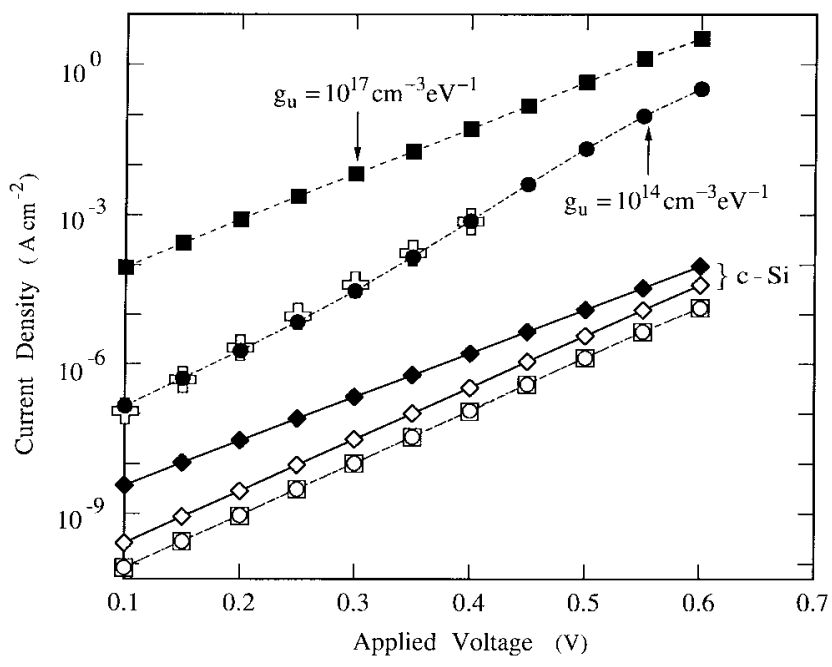

Fig. 7. N-side (full symbol) and P-side (empty symbol) recombination current density versus the applied voltage for a c-Si $(\diamond) \mathrm{PN}$ homojunction diode and an a-Si:H(N)/c-Si(P) $(\square, \bigcirc)$ heterojunction diode. Experimental data (ß) are from [29].

current, via the quasi-Fermi level discontinuity (10b). The values used for the calculation are $\mathrm{N}_{A}=1 \times 10^{16} \mathrm{~cm}^{-3}$, $\mathrm{N}_{D}=7 \times 10^{15} \mathrm{~cm}^{-3}, \mathrm{n}_{i}(\mathrm{a}-\mathrm{Si}: \mathrm{H})=5 \times 10^{5} \mathrm{~cm}^{-3}[22]$ and $\tau(\mathrm{c}-\mathrm{Si})=5 \mu \mathrm{s}$ [24]. In the analysis, we have modeled the bulk trap distribution in amorphous silicon as a combination of uniform and exponential profiles. For the sake of simplicity, and with no loss of generality, Gaussian profiles have not been taken into account [24], [25]. Experimental values for the trap band tail were $g_{u}=1 \times 10^{14} \mathrm{~cm}^{-3} \mathrm{eV}^{-1}, T_{A}=T_{D}=450 \mathrm{~K}$, $g_{a}=g_{d}=1 \times 10^{20} \mathrm{~cm}^{-3} \mathrm{eV}^{-1}$ [26]. Moreover, an increase in the uniform density of states, $g_{u}=1 \times 10^{17} \mathrm{~cm}^{-3} \mathrm{eV}^{-1}$, does not modify the P-side current and increases the $\mathrm{N}$-side recombination current [8]. Typical values for the cross section $\sigma_{\mathrm{n}}=\sigma_{\mathrm{p}}=5 \times 10^{-15} \mathrm{~cm}^{2}$ have been fixed [22]. Finally, interface states in amorphous material, $\mathrm{N}_{i t}=2 \times 10^{11} \mathrm{~cm}^{-2}$, are taken into account through the effective built-in potential [27], [28]. Results are in good agreement with experimental data previously reported [29].

Once the materials have been selected, the designer is only free to change the $\mathrm{N}$-side and the P-side doping density. Applying the analysis described in Section III, we studied the influence of the doping concentration on the $I-V$ characteristic and obtained a general rule which enables $(\mathrm{N}) \mathrm{a}-\mathrm{Si}: \mathrm{H} /(\mathrm{P}) \mathrm{c}-\mathrm{Si}$ heterojunction diodes with minimum total space charge recombination currents to be designed. This analysis is also useful for optoelectronics and heterojunction bipolar devices, and in general the conclusions are valid for noncrystalline/crystalline junctions.

Fig. 8 shows the total $J_{\mathrm{SRH}}$ for several $\mathrm{N}_{A}, \mathrm{~N}_{D}$ doping concentrations, and the other values are the same as in Fig. 7. As in the analysis above, the total recombination current arises from the lowest doped SCR side when doping levels are asymmetrical, whether it is crystalline or noncrystalline. The minimum value of the achievable total current becomes saturated, when the recombination current arises from the crystalline side. On the other hand, when the N-side is slightly more doped than the P-side, there is a change in the slope of 


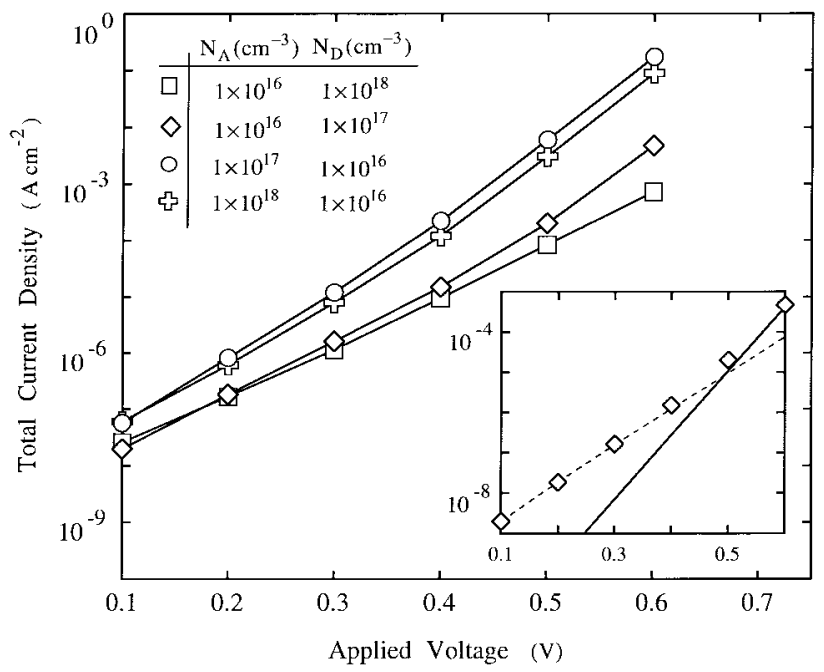

Fig. 8. Total recombination current density of an a-Si:H(N)/c-Si(P) heterojunction diode for several $\mathrm{N}_{A}, \mathrm{~N}_{D}$ values. Inset: for $\mathrm{N}_{A}=1 \times 10^{16} \mathrm{~cm}^{-3}$ and $\mathrm{N}_{D}=1 \times 10^{17} \mathrm{~cm}^{-3}$, the total current is shown together with depicted the currents arising from the amorphous (-) and crystalline (- -) sides.

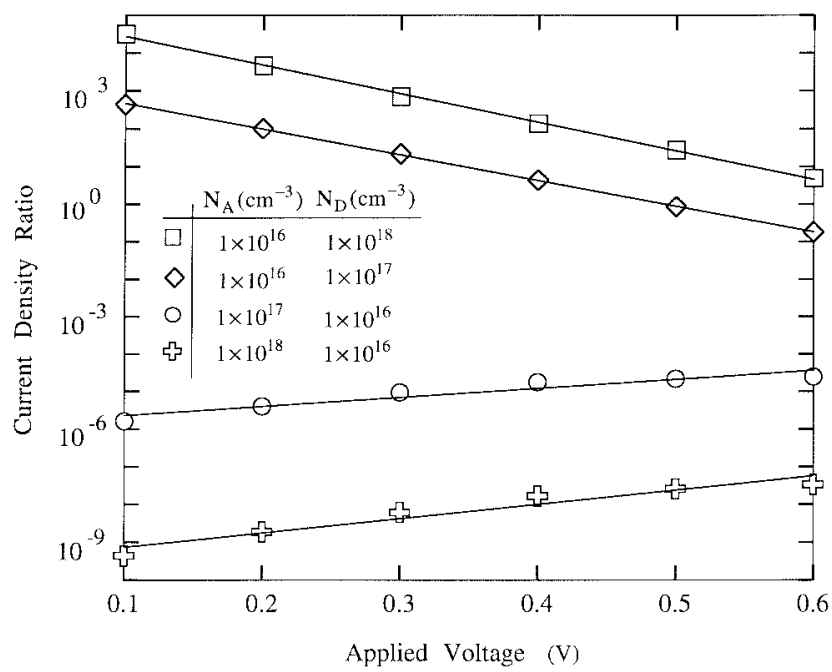

Fig. 9. P-side/N-side recombination current ratio versus the applied voltage for an a-Si: $\mathrm{H}(\mathrm{N}) / c-\mathrm{Si}(\mathrm{P})$ heterojunction diode for several $\mathrm{N}_{A}, \mathrm{~N}_{D}$ values.

the $I-V$ characteristic. This is due to the fact that the current arising from the amorphous side with a lower ideality factor becomes dominant at high voltages.

Just as for the analysis of crystalline devices in Section III, we were able to establish the ratio of the recombination current arising from the $\mathrm{P}$ - and $\mathrm{N}$-sides (see Fig. 9). From the figure, we conclude that the recombination current at the $\mathrm{N}$-side may be several orders of magnitude smaller than the recombination at the P-side, despite the presence of the density of states in the amorphous material, if it is more doped. However, it should be noted that the ratio value depends on the voltage applied because both recombination currents have different ideality factors.

The following general rule is obtained: if the additional recombination current, introduced at the N-side of the SCR because a noncrystalline material is used, is to be minimized, the doping concentration of the noncrystalline side has to be higher than the crystalline side. This is a severe restriction on HBT applications: although the recombination current can be minimized and, consequently, the current gain considerably increased (useful for analog applications) simultaneous high cutoff frequency can not be achieved. In fact, one of the advantages of using heterojunctions at the emitter-base in bipolar transistors is that the base can be highly doped with no current gain degradation. This high base doping leads to small values of the base resistance and enhances the frequency performances. To circumvent this drawback, several possibilities are currently being explored. One of them is to reduce the recombination current at the $\mathrm{N}$-side of the junction by using microcrystalline materials which reduce the density of states inside the forbidden band [4], [6], while enhancing the conductivity of the layer. Alternatively, the non ideal characteristics of the base current can be removed, as has been pointed out by Kondo et al. [5], by shifting the P-N junction from the heterointerface by forming a very thin $\mathrm{N}$-type layer below the heteroemitter layer.

\section{CONCLUSIONS}

A general formulation of the SRH recombination currents in the SCR of a heterojunction has been developed. The doping ratio and the discontinuities both in the bandgap and the conduction band have been identified as the variables which determine the quotient of the recombination currents originated at each side of the SCR. Consequently, by properly choosing the ratio between the doping concentrations on both sides of the junction, the origin of the measured recombination current may be chosen. This is mainly useful in junctions with highly asymmetrical lifetimes and in particular in noncrystalline/crystalline heterojunctions.

Noncrystalline/crystalline heterojunctions have been analyzed as potential candidates for providing injector junctions in wide bandgap emitter silicon HBT. The analysis shows that, in order to minimize the SCR recombination current, the doping concentration in the noncrystalline side should be higher than in the crystalline side. This limits their application to high current gain and low frequency devices. As a possible solution to this drawback, the density of states in the noncrystalline material can be reduced by using microcrystalline instead of amorphous materials or the $\mathrm{P} / \mathrm{N}$ junction can be removed from the heterointerface.

\section{ACKNOWLEDGMENT}

The authors thank A. Garijo for his support throughout.

\section{REFERENCES}

[1] J. Symons, J. Nijs, and R. P. Mertens, "The emitter-base interface current in silicon bipolar transistors with emitters deposited by plasmaenhanced CVD," IEEE Trans. Electron Devices, vol. 36, pp. 2889-2894, 1989.

[2] P. Li, Y. Q. Li, and C. A. T. Salama, "A heterojunction bipolar transistor with a thin $\alpha$-Si emitter," IEEE Trans. Electron Devices, vol. 41, pp. 932-935, 1994.

[3] K. Sasaki, M. M. Rahman, and S. Furukawa, "An amorphous SiC:H emitter heterojunction bipolar transistor," IEEE Electron. Device Lett., vol. EDL-6, pp. 311-312, 1985

[4] K. Sasaki, T. Miyajima, Y. Kubota, and S. Furukawa, "Geniune widebandgap microcrystalline emitter Si-HBT with enhanced current gain by 
suppressing homocrystallization," IEEE Trans. Electron Devices, vol 39, pp. 2132-2137, Sept. 1992.

[5] M. Kondo, T. Shiba, Y. Tamaki, and T. Nakamura, "Double layer $\mu \mathrm{c}-$ $\mathrm{Si} / \mathrm{a}-\mathrm{SiC}_{x}$ emitter in a silicon heterojunction bipolar transistor with a cutoff frequency of $47 \mathrm{GHz}, " J$. Electrochem. Soc., vol. 143, pp. $1949-1955,1996$

[6] M. Kuwagaki, K. Imai, and Y. Amemiya, "Properties of high heatresistance $\mu c-\mathrm{SiC}_{x}: \mathrm{H}$ emitter silicon HBT's," Jpn. J. Appl. Phys., vol. 28, pp. L754-L756, 1989.

[7] A. Mittiga, P. Fiorini, M. Falconieri, and F. Evangelisti, "Dark $J-V$ characteristic of p-i-n a-Si:H solar cell," J. Appl. Phys., vol. 66, pp. 2667-2674, 1989.

[8] J. Pallarès, L. F. Marsal, X. Correig, J. Calderer, and R. Alcubilla, "Space charge recombination in $\mathrm{P} / \mathrm{N}$ junctions with a discrete and continuous trap distribution," Solid-State Electron., vol. 41, pp. 17-23, 1997.

[9] C. T. Sah, R. N. Noyce, and W. Shockley, "Carrier generation and recombination in p-n junction characteristics," Proc. IRE, vol. 45, p. 1228, 1957.

[10] W. Shockley and W. T. Read, "Statistics of the recombination of electrons and holes," Phys. Rev., vol. 87, p. 835, 1952.

[11] R. N. Hall, "Electron-hole recombination in germanium," Phys. Rev., vol. 87 , p. $387,1952$.

[12] S. C. Choo, "Carrier generation-recombination in the space charge region of an asymmetrical p-n junction," Solid-State Electron., vol. 11, pp. 1069-1077, 1968.

[13] S. Searles and D. L. Pulfrey, "An analysis of space-charge-region recombination in HBT's," IEEE Trans. Electron Devices, vol. 41, pp. 476-483, 1994.

[14] A. A. Grinberg and S. Luryi, "On the thermionic-diffusion theory of minority transport in heterostructure bipolar transistors," IEEE Trans. Electron Devices, vol. 40, pp. 859-866, 1993.

[15] J. Schneider, E. Koenig, U. Erben, U. Seiler, and H. Schumacher, "Thermionic diffusion model for abrupt HBT's including self-heating inside the multilayer nonplanar device structure," Solid-State Electron., vol. 39, pp. 377-384, 1996.

[16] S. S. Perlman and D. L. Feucht, "p-n heterojunctions," Solid-State Electron., vol. 7, pp. 911-923, 1964.

[17] A. A. Grinberg, M. S. Shur, R. J. Fisher, and H. Morkoç, "An investigation of the effect of the graded-layers and tunnelling on the performance of AlGaAs/GaAs heterojunction bipolar transistors," IEEE Trans. Electron Devices, vol. ED-31, pp. 1758-1765, 1984.

[18] I. S. Gradshteyn and I. M. Ryzhik, Tables on Integrals, Series, and Products. New York: Academic, 1992.

[19] C. M. S. Ng, P. A. Houston, and H. K. Yow, "Analysis of the temperature dependence of current gain in heterojunction bipolar transistor," IEEE Trans. Electron Devices, vol. 44, pp. 17-24, 1997.

[20] R. Stratton, "Theory of field emission from semiconductors," Phys. Rev., vol. 125 , pp. 67-82, 1962

[21] F. A Padovani and R. Stratton, "Field and thermionic-field emission in Schottky barriers," Solid-State Electron., vol. 9, pp. 695-707, 1966.

[22] A. Solhi, M. Sahnoune, H. Lhermite, and O. Bonnaud, "Numerical simulation and experimental verification of the parameters determining the base current in an a-Si:H/c-Si emitter-base heterojunction bipolar transistor," Solid-State Electron., vol. 35, pp. 1609-1620, 1992.

[23] D. M. Garner and A. J. Amaratunga, "A study of frequency response in silicon heterojunction bipolar transistors with amorphous silicon emitters," IEEE Trans. Electron Devices, vol. 43, pp. 1890-1899, 1996.

[24] F. Rubinelli, S. Albornoz, and R. Buitrago, "Amorphous-crystalline silicon heterojunction: theoretical evaluation of the current terms," SolidState Electron., vol. 32, pp. 813-825, 1989

[25] P. S. Gudem and S. G. Chamberlain, "A realistic trap distribution model for numerical simulation of amorphous silicon thin-film transistors and phototransistors," IEEE Trans. Electron Devices, vol. 42, pp. 1333-1339, 1995.

[26] L. F. Marsal, J. Pallarès, X. Correig, J. Calderer, and R. Alcubilla, "Determination of gap states density in PECVD a-Si:H from amorphouscrystalline heterojunctions," in Proc. 18th IEEE Int. Semicond. Conf., 1995, pp. 111-114.

[27] V. S. Veerasamy, G. A. J. Amaratunga, J. S. Park, H. S. MacKenzie, and W. I. Milne, "Properties of n-type tetrahedral amorphous carbon (ta-C)/p-type crystalline silicon heterojunction diodes," IEEE Trans. Electron Devices, vol. 42, pp. 577-585, 1995.

[28] J. P. Donnelly and A. G. Milnes, "The capacitance of p-n heterojunctions including the effects of interface states," IEEE Trans. Electron Devices, vol. 14, pp. 63-68, 1967.

[29] L. F. Marsal, J. Pallarès, X. Correig, J. Calderer, and R. Alcubilla,
"Electrical characterization of n-amorphous/p-crystalline silicon heterojunctions," J. Appl. Phys., vol. 79, pp. 8493-8497, 1996.

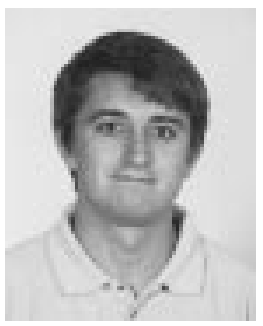

J. Pallarès (S'94) received the degree in physics from the Universitat de Barcelona, Barcelona, Spain, in 1991. Currently, he is pursuing the Ph.D degree at the Universitat Politècnica de Catalunya, Barcelona.

Since 1991, he has been an Assistant Professor in the Electronics Engineering Department, Universitat Rovira i Virgili, Tarragona, Spain. His research interests include the modeling, simulation, and characterization of heterostructure bipolar devices.

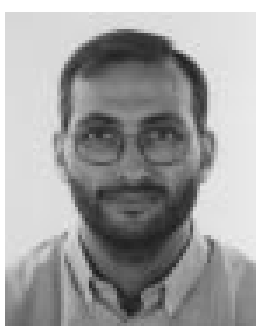

L. F. Marsal (S'94) received the degree in physics from the Universitat de Barcelona, Barcelona, Spain, in 1991. Currently, he is pursuing the Ph.D degree at the Universitat Politècnica de Catalunya, Barcelona.

Since 1991, he has been an Assistant Professor in the Electronics Engineering Department, Universitat Rovira i Virgili, Tarragona, Spain. His research interests include the modeling and characterization of noncrystalline/crystalline heterojunction devices.

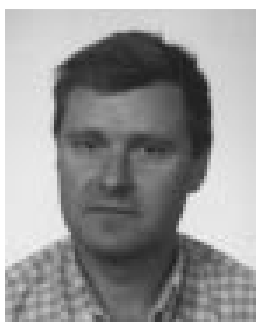

X. Correig received the degree in telecommunication engineering in 1984, and the Ph.D. degree in telecommunication engineering in 1988, from the Universitat Politècnica de Catalunya, Barcelona, Spain.

Currently, he is an Associate Professor in the Electronics Engineering Department, Universitat Rovira i Virgili, Tarragona, Spain. His research interests are in heterojunction semiconductor devices and chemical solid-state gas sensors.

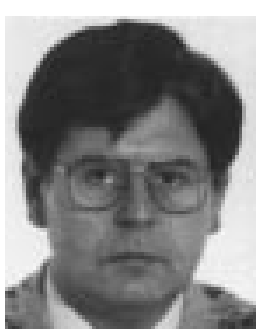

J. Calderer was born in Berga, Catalonia, Spain. He received the degree in physics from the Universitat de Barcelona, Barcelona, Spain, in 1973, and the $\mathrm{Ph} . \mathrm{D}$. degrees from the Universities de Montpellier Montpellier, France, in 1979, and the Universitat de Barcelona in 1981

In 1981, he became an Associate Professor in the Electronic Engineering Department, Universitat Politècnica de Catalunya, Barcelona. His research interests include photovoltaic cells and other bipolar devices. He is currently involved in the study of electronic materials and technologies and their applications to physical and chemical sensors.

R. Alcubilla (M'93) received the Ingeniero de Telecommunicacion degree from the Universitat Politècnica de Catalunya, Barcelona, Spain, in 1981, and the Docteur Ingenieur degree from Universite Paul Sabatier, Toulouse, France, in 1985.

From 1982 to 1985, he worked on GaAs solar cells development at the Laboratoire d'Automatique et Analyse de Systemes, Toulouse. In 1985, he joined the Department of Electronic Engineering, Universitat Politècnica de Catalunya, Barcelona, where he became an Associate Professor in 1987 and a Professor in 1996. His research activities are mainly devoted to the study of new materials for silicon HBT's and sensors. 